\title{
Evaluación de la carga y fatiga mental en docentes. caso de la Universidad Técnica de Ambato.
}

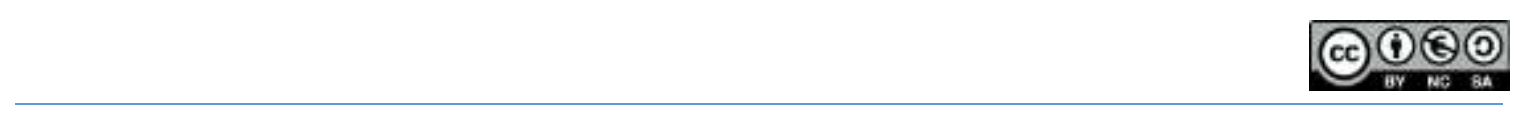

Load evaluation and mental fatigue in teachers. Case of the Technical University of Ambato.

Tania Alexandra Rea Hinojosa ${ }^{1}$., Manolo Alexander Córdova Suárez ${ }^{2}$., Paulina del Roció Gordón Villalba ${ }^{3}$., José Geovanny Vega Pérez ${ }^{4}$., Oscar Eduardo Ruíz Robalino ${ }^{5}$. \& Edwin Leonardo Sánchez Almeida ${ }^{6}$.

\begin{abstract}
DOI: https://doi.org/10.33262/cienciadigital.v9i2.388

Mental Burden was studied in the teaching staff of the Faculty of Food Science and Engineering (FCIAL) Career of Biochemical Engineering of the Technical University of Ambato (UTA) using the NASA-Task Load Index (TLX) method. The mental requirement, physical requirement, time requirement, effort, performance and level of frustration in the teaching staff of the FCIAL was quantified as a psychosocial risk factor and its effects as a result of discomfort due to normal fatigue and chronic fatigue following the NTP standard. 544 applying standardized methods that determine the characteristics of the task and its execution conditions in a single summative result.
\end{abstract}

Methods: The study begins with the identification of individuals exposed to psychosocial risk factors at a critical level using a triple-criteria occupational risk matrix. The NASA-Task Load Index (TLX) method was applied to the exposure group to quantify: The effort, the mental Demand, the Physical Demand, the Temporary Demand, the Performance and the Frustration Level and to determine a final value of calculated mental Load. The application of the method was carried out in two phases: the first one of weighting and the next one of scoring the perception of the subjective sensation of load. The study considers the application

\footnotetext{
${ }^{1}$ Faculty of Health Sciences, Technical University of Ambato, Ambato-Ecuador, ta.rea@uta.edu.ec

2 G+ Energy-Risks \& Engineering Group, Department of Food Science and Engineering, Technical University of Ambato, Ambato-Ecuador, ma.cordova@uta.edu.ec

${ }^{3}$ Faculty of Health Sciences, Technical University of Ambato, Ambato-Ecuador, pdr.gordon@uta.edu.ec

${ }^{4}$ G+ Energy-Risks \& Engineering Group, Department of Food Science and Engineering, Technical University of Ambato, Ambato-Ecuador,jg.vega@uta.edu.ec

5 G+ Energy-Risks \& Engineering Group, Department of Food Science and Engineering, Technical University of Ambato, Ambato-Ecuador, oe.ruíz@uta.edu.ec

${ }^{6}$ Indoamerica Technologic University, Ambato.
} 
of the method in two fortnights. The study is completed by estimating the application of a validated questionnaire to evaluate normal and chronic mental fatigue.

Results: From the risk matrix, $68 \%$ of people exposed to mental load were identified. It was found that in the first fortnight the presence of mental load exceeds $45 \%$ and in the second fortnight it exceeds $69 \%$ of mental load. Regarding mental fatigue for the first fortnight fatigue values are: fatigue $33 \%$; irritability $17 \%$; organization and design of the job $13 \% ; 11 \%$ anxiety; insomnia $8 \%$; loss of attention $6 \%$, dizziness $6 \%$, low performance $6 \%$ for the second half of the year, we observe values of: loss of attention $16 \%$, dizziness $16 \%$, low performance $16 \%$; insomnia $15 \%$; anxiety $14 \%$, fatigue $12 \%$, irritability $10 \%$, organization and design of the job $1 \%$.

Conclution: The statistical results made with the Chi square determines a direct incidence of the mental load with the mental fatigue, being the second fortnight of evaluation the one that has higher values of the level of affectation in mental load. In addition, a greater presence of normal fatigue was observed than chronic fatigue. The evaluated individuals are working more than two consecutive semesters in an 8-hour day in the sector of the Ecuadorian highlands.

Keywords: Psychosocial Risk, Mental Burden, Mental Fatigue.

\section{Resumen}

Se estudió la Carga Mental en el personal docente de la Facultad de Ciencia e Ingeniería en Alimentos (FCIAL) Carrera de Ingeniería Bioquímica de la Universidad Técnica de Ambato (UTA) utilizando el método el NASA-Task Load Index (TLX). Se cuantificó la exigencia mental, exigencia física, exigencia temporal, el esfuerzo, rendimiento y el nivel de frustración en el personal docente de la FCIAL como factor de riesgo psicosocial y sus efectos como resultado de molestias por fatiga Normal y Fatiga Crónica siguiendo la Norma NTP 544 aplicando métodos estandarizados que determinan las características de la tarea y sus condiciones de ejecución en un solo resultado sumativo.

Métodos: El estudio inicia con la identificación de los individuos expuestos a factores de riesgo psicosocial de nivel crítico utilizando una matriz de riesgos laborales de triple criterio. Al grupo de exposición se aplicó el método NASATask Load Index (TLX) para cuantificar: El esfuerzo, la Demanda mental, la Demanda física, la Demanda temporal, el Rendimiento y el Nivel de frustración y determinar un valor final de Carga mental calculado. La aplicación del método se realizó en dos fases: la primera de ponderación y una siguiente de puntuación de la percepción de la sensación subjetiva de carga. El estudio considera la aplicación del método en dos quincenas. El estudio se completa estimando la aplicación de un cuestionario validado para evaluar la fatiga mental normal y crónica. 
Resultados: De la matriz de riesgo se identificó un 68\% de personas expuestas a carga mental. Se encontró que en la primera quincena la presencia de carga mental supera el $45 \%$ y en la segunda quincena supera el $69 \%$ de carga mental. En cuanto a la fatiga mental para la primera quincena los valores de fatiga son de: cansancio $33 \%$; irritabilidad 17\%; organización y diseño del puesto de trabajo 13\%; ansiedad $11 \%$; insomnio $8 \%$; perdida de atención $6 \%$, mareos $6 \%$, bajo rendimiento $6 \%$ para la segunda quincena se observa valores de: perdida de atención 16\%, mareos $16 \%$, bajo rendimiento 16\%; insomnio 15\%; ansiedad 14\%, cansancio $12 \%$, irritabilidad $10 \%$, organización y diseño del puesto de trabajo $1 \%$.

Conclusión: Los resultados estadísticos efectuados con el Chi cuadrado determina una incidencia directa de la carga mental con la fatiga mental, siendo la segunda quincena de evaluación la que tiene valores más elevados del nivel de afectación en carga mental. Además, se comprobó una mayor presencia de Fatiga normal que la Fatiga crónica. Los individuos evaluados se encuentran laborando más de dos semestres consecutivos en jornada de 8 horas en el sector de la sierra ecuatoriana.

Palabras clave: Riesgo Psicosocial, Carga mental, Fatiga Mental.

\section{Introducción.}

Las actividades de enseñanza aprendizaje se caracterizada por los niveles de agotamiento físico y agotamiento emocional (Chang, 2009). Las exigencias físicas y cognitivas en los centros Educativos pueden ocasionar problemas en su salud (González, 2008) específicamente fatiga (Koenig et al., 2018).

Por una disminuida realización personal y falta de apoyo en el trabajo los docentes se sienten descontentos e insatisfechos con sus resultados laborales bajando su rendimiento significativamente (Marrau, 2004) aumentando el índice de ausentismo de corta duración al cansancio y fatiga laboral (Lozada, 2005).

Los programas de atenuación de riesgos psicosocial dependen de la evaluación específica de los factores considerados como críticos en la etapa de identificación inicial (Romero, 2004). Siempre que se determine los elementos específicos que causan el peligro la intervención en los afectados resulta más efectiva y las afecciones tienden a disminuir progresivamente (Solé y Balduque, 2006).

Los métodos de evaluación de riesgos son diversos y dependen de los elementos específicos a evaluarse, la mayoría de instrumentos de evaluación de carga mental considera seis dimensiones definidas. La medición de la carga mental considera la ejecución de la tarea, el procesamiento de la información, mapas mentales, aspectos psicofisiológicos y distribución de recursos en los procesos mentales (Pons y Puig, 2004).

Los procedimientos subjetivos multidimensionales se utilizan recurrentemente, por la diferenciación de las causas de carga mental y las características psicométricas. Entre los métodos subjetivos multidimensionales más destacados y usados está el NASA-Task 
Load Index (TLX) (Díaz Ramiro et al., 2010).

Este trabajo contempla la determinación de los factores preponderantes de carga mental en las afecciones agudas o crónicas de la fatiga en los docentes de la FCIAL de la UTA.

\section{Materiales y método}

\section{Evaluación de la carga mental}

La carga mental se ha definido considerando dos puntos de vista, el primero relacionado a las exigencias de la tarea y el segundo considerando las exigencias de la tarea y las capacidades o recursos de la persona (Pons y Puig, 2004), siendo el segundo enfoque el más aceptado (Karwowski, 2006). Por otro lado, el concepto multidimensional de carga mental agrupa tres elementos principales: la gestión de tiempos, recursos que demanda la tarea y la naturaleza emocional asociada a efectos como la frustración, estrés (Hart y Staveland, 1988). De los métodos para evaluar la carga mental destacan los subjetivos por los altos niveles de sensibilidad, fácil comprensión por parte de los trabajadores, intrusión nula y validez. Estos métodos facilitan establecer diferencias entre las causas manteniendo las tipologías psicométricas (Salvendy, 2012). La variedad de métodos el interminable sin embargo destaca el NASA-Task Load Index (TLX). (Hart y Staveland, 1988), los estudios de fiabilidad y sensibilidad del método indican valores superiores a 0,8 del coeficiente de alpha de Cronbach garantizando su uso (Xiao et al., 2005).

La técnica NASA-Task Load Index (TLX)evalúa seis dimensiones: Esfuerzo, Demanda mental, Demanda física, Demanda temporal, Rendimiento y Nivel de frustración, cuya combinatoria dará un valor final

Se aplica en dos fases. La primera utiliza un instrumento del método que resulta la importancia que manifiesta el individuo de cada una de las seis dimensiones y sus 15 comparaciones de pares con elección de valores de 0 y 1 para tener un valor ponderado como primera aproximación de la opción que considera de mayor importancia ver tabla1.

Tabla 1. Ponderación dimensiones de carga mental

\begin{tabular}{|c|c|c|c|c|c|}
\hline Dimensión 1 & \multicolumn{2}{|c|}{ Valor a contestar } & Dimensión 2 & \multicolumn{2}{|c|}{ Valor a contestar } \\
\hline Exigencia mental & SI & \begin{tabular}{l|l}
$\mathrm{NO}$ & \\
\end{tabular} & Exigencia física & SI & $\mathrm{NO}$ \\
\hline Exigencia mental & \begin{tabular}{|l} 
SI \\
\end{tabular} & \begin{tabular}{l|l|} 
NO & \\
\end{tabular} & Exigencia temporal & SI & $\mathrm{NO}$ \\
\hline Exigencia mental & $\mathrm{SI}$ & $\overline{\mathrm{NO}}$ & Esfuerzo & SI & $\mathrm{NO}$ \\
\hline Exigencia mental & \begin{tabular}{|l|l} 
SI \\
\end{tabular} & $\mathrm{NO}$ & Rendimiento & SI & $\mathrm{NO}$ \\
\hline Exigencia mental & SI & $\mathrm{NO}$ & Frustración & SI & $\mathrm{NO}$ \\
\hline Exigencia física & SI & $\mathrm{NO}$ & Exigencia temporal & SI & $\mathrm{NO}$ \\
\hline Exigencia física & $\mathrm{SI}$ & NO & Esfuerzo & SI & $\mathrm{NO}$ \\
\hline Exigencia física & \begin{tabular}{|l|}
$\mathrm{SI}$ \\
\end{tabular} & $\mathrm{NO}$ & Rendimiento & SI & $\mathrm{NO}$ \\
\hline Exigencia física & \begin{tabular}{|l} 
SI \\
\end{tabular} & $\mathrm{NO}$ & Frustración & SI & $\mathrm{NO}$ \\
\hline Exigencia temporal & \begin{tabular}{|l|} 
SI \\
\end{tabular} & $\mathrm{NO}$ & Esfuerzo & SI & $\mathrm{NO}$ \\
\hline Exigencia temporal & $\mathrm{SI}$ & \begin{tabular}{l|l} 
NO & \\
\end{tabular} & Rendimiento & SI & $\mathrm{NO}$ \\
\hline
\end{tabular}




\begin{tabular}{|c|c|c|c|c|c|}
\hline Exigencia temporal & \begin{tabular}{|l|} 
SI \\
\end{tabular} & \begin{tabular}{l|l}
$\mathrm{NO}$ & \\
\end{tabular} & Frustración & SI & $\mathrm{NO}$ \\
\hline Esfuerzo & SI & \begin{tabular}{l|l} 
NO & \\
\end{tabular} & Rendimiento & SI & $\mathrm{NO}$ \\
\hline Esfuerzo & \begin{tabular}{|l|} 
SI \\
\end{tabular} & \begin{tabular}{l|l|} 
NO & \\
\end{tabular} & Frustración & SI & $\mathrm{NO}$ \\
\hline Rendimiento & \begin{tabular}{|l|} 
SI \\
\end{tabular} & \begin{tabular}{l|l} 
NO & \\
\end{tabular} & Frustración & SI & NO \\
\hline
\end{tabular}

Nota: Interacción de las dimensiones. Si escoge el valor SI en la primera dimensión la segunda es lo contrario. Se presenta 5 interacciones para cada variable. Adaptado de (de Arquer y Nogareda, 2000).

En la segunda fase se utiliza una plantilla del método para que el individuo escoja el valor de una escala de 0 a 100 que el estime para cada una de las seis dimensiones de la carga mental ver tabla 2 .

Una vez que se definieron las personas expuestas y dispuestos a colaborar, el equipo investigador realizó una socialización del método y haciendo referencia de una participación voluntaria y anónima. Luego de obtener las plantillas llenas con las respuestas de cada participante se desarrolló el informe respectivo para que conozca la unidad competente en Seguridad y Salud en el trabajo.

Tabla 2. Modelo de encuesta de carga mental

\begin{tabular}{|c|c|c|c|c|c|c|c|c|c|c|c|c|}
\hline N. & \multicolumn{2}{|c|}{ PREGUNTAS } & \multicolumn{10}{|c|}{ RESPUESTAS } \\
\hline \multirow{3}{*}{1} & \multirow{3}{*}{ EXIGENCIA MENTAL } & \multirow{3}{*}{$\begin{array}{l}\text { ¿Siente que en su trabajo } \\
\text { tiene muchas actividades } \\
\text { relacionadas con pensar, } \\
\text { calcular, recordar, decidir? }\end{array}$} & \multicolumn{3}{|c|}{ Nunca } & \multicolumn{3}{|c|}{ a veces } & \multicolumn{4}{|c|}{ siempre } \\
\hline & & & 10 & 20 & 30 & 40 & 50 & 60 & 70 & 80 & 90 & 100 \\
\hline & & & & & & & & & & & & \\
\hline \multirow{3}{*}{2} & \multirow{3}{*}{ EXIGENCIA FÍSICA } & \multirow{3}{*}{$\begin{array}{c}\text { ¿Cuánta actividad física } \\
\text { desarrolla en sus } \\
\text { actividades? }\end{array}$} & \multicolumn{3}{|c|}{ Baja } & \multicolumn{3}{|c|}{ media } & \multicolumn{4}{|c|}{ alta } \\
\hline & & & 10 & 20 & 30 & 40 & 50 & 60 & 70 & 80 & 90 & 100 \\
\hline & & & & & & & & & & & & \\
\hline \multirow{4}{*}{3} & \multirow{4}{*}{ EXIGENCIA TEMPORAL } & \multirow{4}{*}{$\begin{array}{c}\text { ¿Siente presión de } \\
\text { tiempo debido al ritmo } \\
\text { al cual se sucedían las } \\
\text { tareas o los elementos } \\
\text { de las tareas? }\end{array}$} & \multicolumn{3}{|c|}{ Nunca } & \multicolumn{3}{|c|}{ a veces } & \multicolumn{4}{|c|}{ siempre } \\
\hline & & & 10 & 20 & 30 & 40 & 50 & 60 & 70 & 80 & 90 & 100 \\
\hline & & & & & & & & & & & & \\
\hline & & & & & & & & & & & & \\
\hline \multirow{4}{*}{4} & \multirow{4}{*}{ ESFUERZO } & \multirow{4}{*}{$\begin{array}{c}\text { ¿En qué medida ha } \\
\text { tenido que trabajar } \\
\text { (física o mental mente) } \\
\text { para alcanzar su nivel } \\
\text { de resultado? }\end{array}$} & \multicolumn{3}{|c|}{ Baja } & \multicolumn{3}{|c|}{ media } & \multicolumn{4}{|c|}{ alta } \\
\hline & & & 10 & 20 & 30 & 40 & 50 & 60 & 70 & 80 & 90 & 100 \\
\hline & & & & & & & & & & & & \\
\hline & & & & & & & & & & & & \\
\hline \multirow{4}{*}{5} & \multirow{4}{*}{ RENDIMIENTO } & \multirow{4}{*}{$\begin{array}{l}\text { ¿Hasta qué punto cree } \\
\text { que ha tenido éxito en } \\
\text { los objetivos } \\
\text { establecidos en sus } \\
\text { actividades? }\end{array}$} & \multicolumn{3}{|c|}{ Bueno } & \multicolumn{3}{|c|}{ medio } & \multicolumn{4}{|c|}{ malo } \\
\hline & & & 10 & 20 & 30 & 40 & 50 & 60 & 70 & 80 & 90 & 100 \\
\hline & & & & & & & & & & & & \\
\hline & & & & & & & & & & & & \\
\hline & & $\begin{array}{l}\text { ¿Durante la tarea, en } \\
\text { qué medida se ha }\end{array}$ & Nunc & & & & veces & & & siem & & \\
\hline 6 & FRUSTRACIÓN & sentido inseguro, & 10 & 20 & 30 & 40 & 50 & 60 & 70 & 80 & 90 & 100 \\
\hline & & desalentado, irritado, & & & & & & & & & & \\
\hline
\end{tabular}

Nota: Ficha de evaluación de carga mental. Adaptado de (SOUSA, 2018)

\section{Fatiga Mental}

Las consecuencias de la carga mental están relacionadas con la fatiga; el problema aparece cuando esta recuperación no se hace posible produciéndose la fatiga crónica que, en muchos casos, puede incidir en un aumento del índice de absentismo (Noriega et al., 2000).

La fatiga mental puede derivar en: menor atención, síntomas de cansancio, menor 
rendimiento, irritabilidad, ansiedad, insomnio, mareos (Duarte et al., 2019). Para este trabajo se ha tomado en cuenta los efectos de la fatiga en un cuestionario de aplicación directa (Cárdenas et al., 2008) como se observa en la plantilla de preguntas de carga mental de la tabla 3.

Tabla 3. Modelo de encuesta de fatiga mental

\begin{tabular}{|c|c|c|}
\hline N. & $\begin{array}{r}\text { PREGUNTAS } \\
\end{array}$ & RESPUESTAS \\
\hline 1 & ¿Siente que ha bajado su rendimiento últimamente? & \begin{tabular}{|l|l|l|l|} 
SI & & NO & \\
\end{tabular} \\
\hline 2 & $\begin{array}{l}\text { ¿Ha sentido síntomas de cansancio cuando está } \\
\text { realizando sus actividades? }\end{array}$ & \begin{tabular}{|l|l|l|l|} 
SI & & NO & \\
\end{tabular} \\
\hline 3 & $\begin{array}{l}\text { ¿Siente que pierde la atención al realizar sus } \\
\text { actividades? }\end{array}$ & \begin{tabular}{|l|l|l|l|} 
SI & & NO & \\
\end{tabular} \\
\hline 4 & ¿Ha sentido síntomas de irritabilidad últimamente? & \begin{tabular}{|l|l|l|l|} 
SI & & NO & \\
\end{tabular} \\
\hline 5 & ¿Ha sentido síntomas de ansiedad últimamente? & \begin{tabular}{|l|l|l|l|} 
SI & & NO & \\
\end{tabular} \\
\hline 6 & ¿Ha sentido insomnio últimamente? & \begin{tabular}{|l|l|l|l|} 
SI & & NO & \\
\end{tabular} \\
\hline 7 & ¿Ha sentido mareos últimamente? & 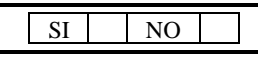 \\
\hline 8 & $\begin{array}{l}\text { ¿Cree usted que la organización de su puesto de trabajo } \\
\text { tiene un buen diseño de acuerdo a sus necesidades? }\end{array}$ & \begin{tabular}{|l|l|l|l|} 
SI & & NO & \\
\end{tabular} \\
\hline
\end{tabular}

Nota: Ficha de evaluación de la fatiga mental. Si corresponde a un valor de 0 y No corresponde a un valor de 1. Adaptado de (Irala, 1975).

\section{Resultados.}

\section{Resultado global de la aplicación del método NASA LTX primera quincena.}

Al finalizar la primera quincena del mes los resultados de la encuesta aplicada siguiendo el método NASA LTX a los 17 trabajadores de la facultad de Ciencia e Ingeniería en Alimentos presenta los siguientes resultados.

Figura 1. Establece los valores ponderados finales de la Carga Mental como resultado de la aplicación del método NASA LTX. Se desarrolla la fundamentación teórica de la investigación.

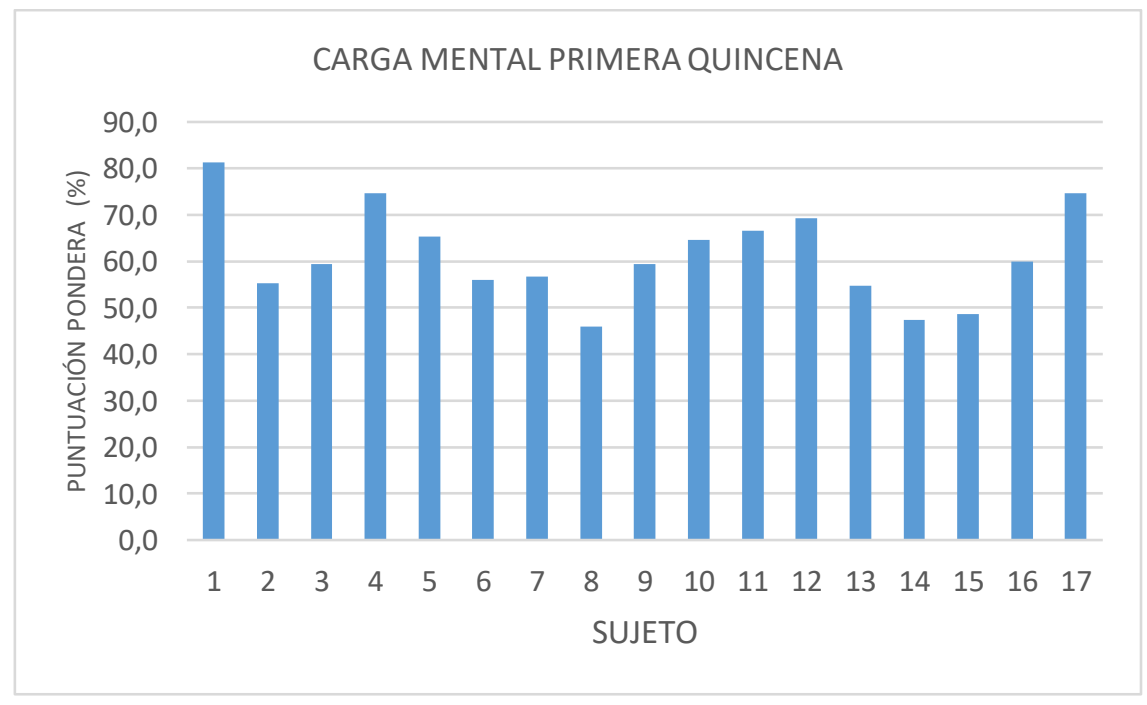




\section{Resultado global de la aplicación del método NASA LTX segunda quincena}

Al finalizar la segunda quincena del mes el resultado de la encuesta aplicada se observa en la figura 2:

Figura 2. Establece los valores ponderados finales de la Carga Mental como resultado de la aplicación del método NASA LTX. Se desarrolla la fundamentación teórica de la investigación.

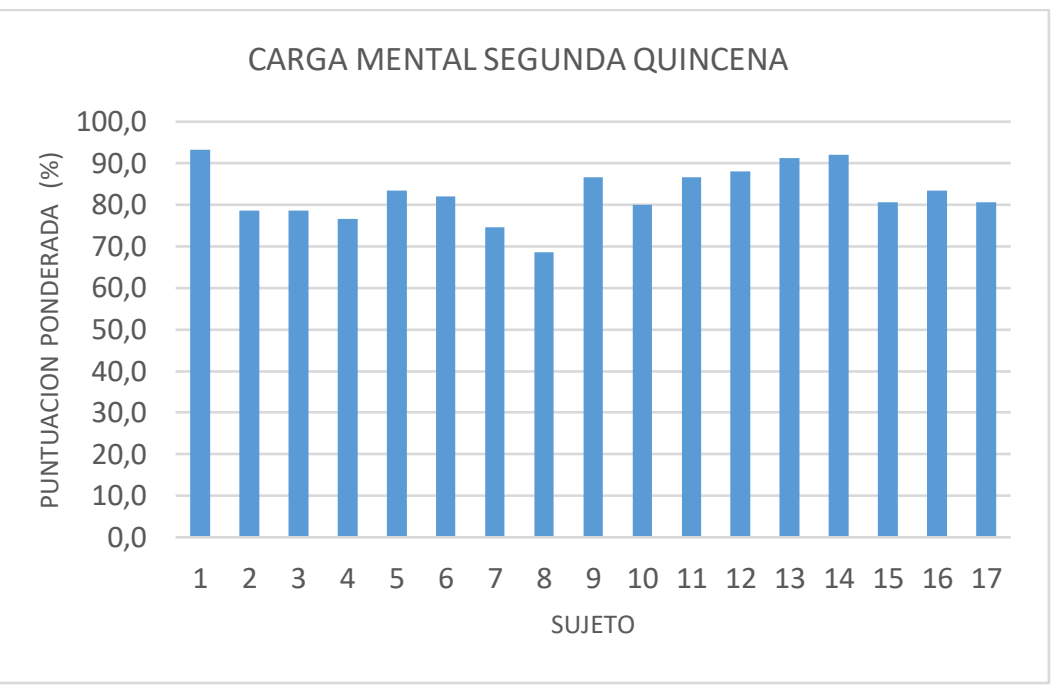

\section{Resultado de la fatiga}

Aplicando la herramienta de evaluación de fatiga mental se obtuvieron los siguientes resultados para la primera semana. Ver figura 3.

Figura 3. Resultados de la fatiga mental aplicado al grupo en la primera quincena 


\section{VALORESDE FATIGA PRIMERA QUINCENA}

¿Siente que ha bajado su rendimiento últimamente?

- ¿Ha sentido síntomas de cansancio cuando está realizando sus actividades?

- ¿Siente que pierde la atención al realizar sus actividades?

¿Ha sentido síntomas de irritabilidad últimamente?

- ¿Ha sentido síntomas de ansiedad últimamente?

¿ ¿Ha sentido insomnio últimamente?

¿ ¿Ha sentido mareos últimamente?

- ¿Cree usted que la organización de su puesto de trabajo tiene un buen diseño de acuerdo a sus necesidades?

Aplicando la herramienta de evaluación de fatiga mental se obtuvieron los siguientes resultados para la segunda semana. Ver figura 4.

Figura 4. Resultados de la fatiga mental aplicado al grupo en la segunde quincena. 


\section{VALORES EN PORCENTAJE DE LA DECISIÓN NO SEGÚN LA PREGUNTA}

¿Siente que ha bajado su rendimiento últimamente?

- ¿Ha sentido síntomas de cansancio cuando está realizando sus actividades?

- ¿Siente que pierde la atención al realizar sus actividades?

¿ ¿Ha sentido síntomas de irritabilidad últimamente?

- ¿Ha sentido síntomas de ansiedad últimamente?

¿ ¿Ha sentido insomnio últimamente?

— ¿Ha sentido mareos últimamente?

- ¿Cree usted que la organización de su puesto de trabajo tiene un buen diseño de acuerdo a sus necesidades?

El valor calculado del Chi Cuadrado $\left(\mathrm{X}^{\wedge} 2 \mathrm{cal}\right)$ con 7 grados de libertad y un nivel de significación del $5 \%$ es de 20,5 por lo consiguiente de conformidad a lo establecido en la regla de decisión del Chi Cuadrado se establece que la carga mental en el personal docente de la Facultad de Ciencia e Ingeniería en Alimentos incide en la fatiga mental.

\section{Discusión.}

Para tener valores más representativos de carga mental en personal docente de la UTA se recomienda incluir un número superior de individuos o realizar en todas las Facultades el mismo análisis.

\section{Conclusiones.}

- Se puede concluir que los trabajadores experimentan mayores valores por carga mental en la segunda semana tendiendo al aumento de porcentaje en el siguiente orden: Sujeto 1 aumenta el $12 \%$, sujeto 2 el $23.3 \%$, sujeto 3 el $19.3 \%$, sujeto 4 el $2 \%$, sujeto 5 el $18 \%$, sujeto 6 el $26 \%$, sujeto 7 el $18 \%$, sujeto 8 el $22.7 \%$, sujeto 9 el $27.3 \%$, sujeto 10 el $15.3 \%$, sujeto 11 el $20 \%$, sujeto 12 el $18.7 \%$, sujeto 13 el $36.7 \%$, sujeto 14 el $44.7 \%$, sujeto 15 el $32 \%$, sujeto 16 el $23.3 \%$ y sujeto 17 el $6 \%$. De la matriz de riesgo se identificó un $68 \%$ de personas expuestas a carga mental. Se encontró que en la primera quincena la presencia de carga mental supera el $45 \%$ y en la segunda quincena supera el $69 \%$ de carga mental.

- Lo valores de fatiga mental para la primera quincena son de: cansancio 33\%; irritabilidad 17\%; organización y diseño del puesto de trabajo 13\%; ansiedad 
$11 \%$; insomnio $8 \%$; perdida de atención $6 \%$, mareos $6 \%$, bajo rendimiento $6 \%$ para la segunda quincena se observa valores de: perdida de atención $16 \%$, mareos $16 \%$, bajo rendimiento $16 \%$; insomnio $15 \%$; ansiedad $14 \%$, cansancio $12 \%$, irritabilidad $10 \%$, organización y diseño del puesto de trabajo $1 \%$.

- Según los resultados con la técnica del CHI CUADRADO se observa un valor de 20,5 por lo que se acepta la hipótesis de que la carga mental a la que se exponen los docentes de la Facultad de Ciencia e Ingeniería en Alimentos, Carrera de Ingeniería Bioquímica incide en la Fatiga Mental.

\section{Referencias Bibliográficas}

Cárdenas, S., Lucas, M., Idoate García, V. M. y Llano Lagares, M. (2008). SOFI-SM: cuestionario para el análisis de la fatiga laboral física, mental y psíquica.

Chang, M. L. (2009). An appraisal perspective of teacher burnout: Examining the emotional work of teachers. Educational Psychology Review, 21(3), 193-218. doi: 10.1007/s10648-009-9106-y

de Arquer, I. y Nogareda, C. (2000). NTP 544: Estimación de la carga mental de trabajo: el método NASA TLX. Madrid: Instituto Nacional de Higiene y Seguridad en el Trabajo, Ministerio de Trabajo y Seguridad Social del Gobierno de Espa na.

Díaz Ramiro, E., Rubio Valdehita, S., Martín García, J. y Luceño Moreno, L. (2010). Estudio psicométrico del índice de carga mental NASA-TLX con una muestra de trabajadores españoles. Revista de Psicología del Trabajo y de las Organizaciones, 26(3), 191-199.

Duarte, R. E., Velasco, E., Sánchez-Sosa, J. J. y Reyes-Lagunes, L. I. (2019). Validación psicométrica de la Escala de gravedad de fatiga en médicos residentes mexicanos. Educación Médica, 20(1), 28-36.

González, N. (2008). Prevalencia del estrés en la satisfacción laboral de los docentes universitarios. REDHECS: Revista electrónica de Humanidades, Educación y Comunicación Social, 3(4), 68-89.

Hart, S. G. y Staveland, L. E. (1988). Development of NASA-TLX (Task Load Index):

Results of empirical and theoretical research Advances in psychology (Vol. 52, pp. 139-183): Elsevier.

Irala, N. (1975). Eficiencia sin fatiga en el trabajo mental (Vol. 12): Editorial SAL TERRAE.

Karwowski, W. (2006). Mental workload: theory, measurement, and application 
International Encyclopedia of Ergonomics and Human Factors-3 Volume Set (pp. 866-869): CRC Press.

Koenig, A., Rodger, S. y Specht, J. (2018). Educator Burnout and Compassion Fatigue:

A Pilot Study. Canadian Journal of School Psychology, 33(4), 259-278. doi: $10.1177 / 0829573516685017$

Lozada, M. A. (2005). La docencia: ¿ un riesgo para la salud? Avances en enfermería, 23(1), 18-30.

Marrau, C. M. (2004). El síndrome de Burnout y sus posibles consecuencias en el trabajador docente. Fundamentos en humanidades(10), 53-68.

Noriega, M., Laurell, C., Martínez, S., Méndez, I. y Villegas, J. (2000). Interacción de las exigencias de trabajo en la generación de sufrimiento mental. Cadernos de Saúde Pública, 16, 1011-1019.

Pons, I. D. y Puig, R. F. (2004). Revisión del concepto de carga mental: evaluación, consecuencias y proceso de normalización. Anuario de psicología/The UB Journal of psychology, 35(4), 521-546.

Romero, J. C. R. (2004). Métodos de evaluación de riesgos laborales: Ediciones Díaz de Santos.

Salvendy, G. (2012). Handbook of human factors and ergonomics: John Wiley \& Sons.

Solé, M. D. y Balduque, M. (2006). El programa de ayuda al empleado (EAP): intervención individual en la prevención de riesgos psicosociales. Madrid: Instituto Nacional de Seguridad e Higiene en el Trabajo.

SOUSA, J. (2018). Avaliação da Carga Mental de Trabalho de Socorristas do Serviço de Atendimento Móvel de Urgência.

Xiao, Y., Wang, Z., Wang, M. y Lan, Y. (2005). The appraisal of reliability and validity of subjective workload assessment technique and NASA-task load index. Zhonghua lao dong wei sheng zhi ye bing za zhi= Zhonghua laodong weisheng zhiyebing zazhi= Chinese journal of industrial hygiene and occupational diseases, 23(3), 178-181.

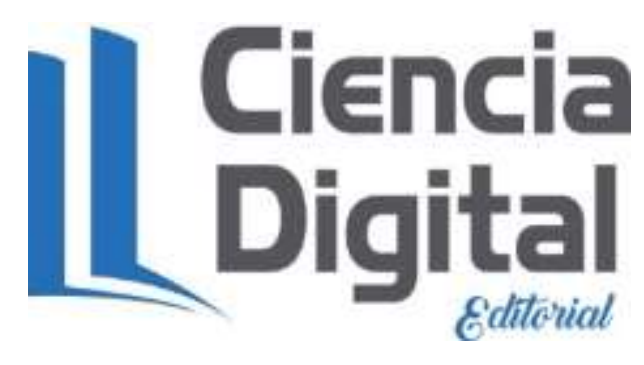




\section{Para citar el artículo indexado.}

Rea T., Córdova M., Gordón P., Vega J., Ruíz O. \& Sánchez E. (2019). Evaluación de la carga y fatiga mental en docentes. caso de la Universidad Técnica de Ambato. Revista electrónica Ciencia Digital 3(2), 252-263. Recuperado desde: http://cienciadigital.org/revistacienciadigital2/index.php/CienciaDigital/article/view/388 $\underline{1836}$

\section{¿Ciencia}

El artículo que se publica es de exclusiva responsabilidad de los autores y no necesariamente reflejan el pensamiento de la Revista Ciencia Digital.

El artículo queda en propiedad de la revista y, por tanto, su publicación parcial y/o total en otro medio tiene que ser autorizado por el director de la Revista Ciencia Digital.
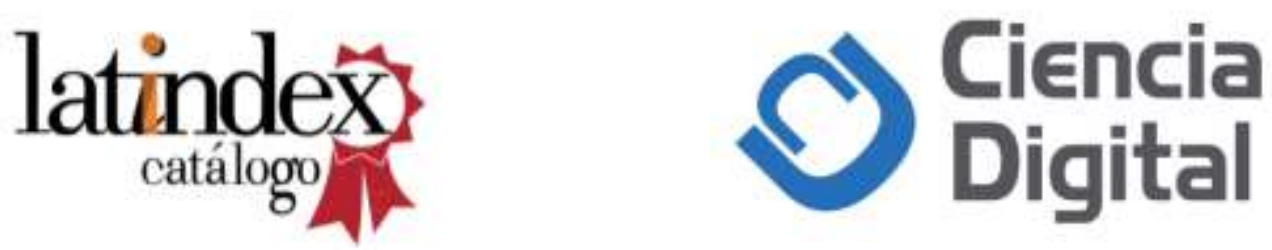